\title{
Identification of stable configurations in the superhydrogenation sequence of polycyclic aromatic hydrocarbon molecules
}

\author{
Pernille A. Jensen, ${ }^{1}$ Mirko Leccese, ${ }^{2}$ Frederik D. S. Simonsen, ${ }^{1}$ Anders W. Skov, ${ }^{1}$ \\ Matteo Bonfanti, ${ }^{2}$ John D. Thrower ${ }^{\oplus},{ }^{1}$ Rocco Martinazzo ${ }^{2}$ and Liv Hornekær ${ }^{1,3 \star}$ \\ ${ }^{1}$ Department of Physics and Astronomy, Aarhus University, Ny Munkegade 120, DK-8000 Aarhus C, Denmark \\ ${ }^{2}$ Department of Chemistry, Universita degli Studi di Milano, Milan, Italy \\ ${ }^{3}$ Interdisciplinatry Nano-Science Centre (iNano), Aarhus University, Denmark
}

Accepted 2019 April 25. Received 2019 April 25; in original form 2019 March 12

\begin{abstract}
Superhydrogenated polycyclic aromatic hydrocarbon (PAH) molecules have been demonstrated to act as catalysts for molecular hydrogen formation under interstellar conditions. Here we present combined thermal desorption mass spectrometry measurements and density functional theory calculations that reveal the most stable configurations in the superhydrogenation sequence of the PAH molecule coronene $\left(\mathrm{C}_{24} \mathrm{H}_{12}\right)$. Specifically, the experiments demonstrate the presence of stable configurations of superhydrogenated coronene at specific hydrogenation levels of 2,10,14, 18, and 24 extra hydrogen atoms. Density functional theory calculations of binding energies and barrier heights explain why these configurations are particularly stable and provide new insights into the superhydrogenation process of PAH molecules under interstellar conditions. Furthermore, an experimental cross-section for the first hydrogen atom addition to the neutral coronene molecule of $\sigma_{\text {add }}=2.7_{-0.9}^{+2.7} \times 10^{-2} \AA^{2}$ is derived from the experimental hydrogenation data.
\end{abstract}

Key words: ISM: clouds - ISM: molecules-Photodissociation region.

\section{INTRODUCTION}

Polycyclic aromatic hydrocarbons (PAHs) are believed to lock up 510 per cent of the carbon available in the interstellar medium (ISM) (Hudgins, Bauschlicher \& Allamandola 2005; Tielens 2013). They are widely accepted to be the carriers of the unidentified infrared bands (Leger \& Puget 1984; Allamandola, Tielens \& Barker 1989a; Allamandola et al. 1989b; Allamandola, Hudgins \& Sandford 1999; Peeters et al. 2002; Tielens 2008), and have been suggested as carriers of some of the diffuse interstellar bands (DIBs) (Léger \& d'Hendecourt 1985; Duley 2006). These studies have shown that the physical, chemical, and charge states of interstellar PAHs vary through interstellar environments. In diffuse cloud environments, PAHs are found to be mainly in the gas phase. In dense clouds however they appear in both the gas phase (Bregman \& Temi 2001) and the condensed phase (Chiar et al. 2000). Studies of PAHs embedded in icy mantles have been performed with emphasis on spectroscopy (Allamandola et al. 1989a; Bernstein, Sandford \& Allamandola 2005; Bauschlicher, Peeters \& Allamandola 2009; Boersma et al. 2010) and solid state photophysics (Bernstein et al. 1999, 2002; Elsila et al. 2006; Bouwman et al. 2009, 2011a,b).

^E-mail: liv@phys.au.dk
These studies reveal that there is potentially a very rich interstellar chemistry involving PAHs, and indeed, when including PAHs in the gas-phase chemical reaction networks in dense clouds, a strong influence on the abundance of many molecular species is found (Wakelam \& Herbst 2008).

In photodissociation regions (PDRs) PAHs are believed to play an important role in the photoelectric heating of the gas, through absorption of stellar far-ultraviolet (FUV) photons (Bakes \& Tielens 1994), which strongly influence the chemistry (Bakes \& Tielens 1998). Furthermore, the interaction between PAHs and atomic hydrogen is of interest, due to the potentially important role PAHs may play in hydrogen formation. Observations of the $\rho$-Oph PDR show a correlation between the PAH and $\mathrm{H}_{2}$ emission (Habart et al. 2003), leading to the proposal that PAHs might catalyse $\mathrm{H}_{2}$ formation in PDRs through addition and abstraction reactions on PAHs and very small grains (VSGs) (Habart et al. 2004).

The formation of $\mathrm{H}_{2}$ in PDRs is still poorly understood. Grain surface reactions involving physisorbed hydrogen atoms have been shown to be efficient at low temperatures in dense and diffuse clouds (Katz et al. 1999; Manico et al. 2001; Hornekær et al. 2003), but they are not sufficiently efficient at the higher temperatures prevalent in PDRs to account for the abundance of $\mathrm{H}_{2}$ observed in those regions. The interaction between PAHs and hydrogen has previously been studied for PAH cations, both theoretically (Cassam-Chenaï, 
Pauzat \& Ellinger 1994; Bauschlicher 1998; Le Page, Snow \& Bierbaum 2009) and experimentally (Boschman et al. 2012; Klærke et al. 2013; Cazaux et al. 2016). These studies showed that it is possible to superhydrogenate $\mathrm{PAH}$ cations, with a clear preference for the addition of an odd number of $\mathrm{H}$ atoms, suggesting that $\mathrm{PAH}$ cations could act as a catalyst for $\mathrm{H}_{2}$ formation. Hydrogenation of PAH cations has been suggested to protect PAHs from photoinduced fragmentation (Reitsma et al. 2014), while other studies suggest that the weakening of the carbon skeleton as a result of $\mathrm{H}$ atom addition might actually lead to hydrogenated PAHs being more prone to fragmentation (Gatchell et al. 2015; Wolf et al. 2016). Sequential hydrogenation has also been demonstrated to lead to fragmentation as a result of the exothermic $\mathrm{C}-\mathrm{H}$ bond formation process, although the low $\mathrm{H}$-atom fluxes present in the ISM make this an unlikely scenario (Rapacioli et al. 2018). The charge state of PAHs in PDRs, however, depends strongly on the FUV flux, and therefore on visual extinction $A_{v}$. At the surface of PDRs, cations are dominant, while further into the cloud neutral PAHs and even anions become important (Bakes \& Tielens 1998). Hydrogenation of neutral PAHs has been studied theoretically, and results suggest that the highest addition barrier is that of the first atom, with a barrier of around $60 \mathrm{meV}$ (Rauls \& Hornekær 2008; Rasmussen, Henkelman \& Hammer 2011), and that abstraction reactions in which $\mathrm{H}_{2}$ is formed are possible. These results have been confirmed by experiments (Mennella et al. 2012; Thrower et al. 2012; Skov, Thrower \& Hornekær 2014; Thrower et al. 2014), which have shown that it is possible to add excess deuterium atoms (at $2300 \mathrm{~K}$ ) to all sites on the coronene molecule, thus forming the fully superhydrogenated species perhydrocoronene $\left(\mathrm{C}_{24} \mathrm{H}_{36}\right)$. Furthermore, the experiments demonstrate catalytic HD formation via exchange of the original edge hydrogen atoms for deuterium through abstraction reactions. PDR models, including PAHs, suggest that abstraction reactions play a role in $\mathrm{H}_{2}$ formation in more shielded regions (Andrews, Candian \& Tielens 2016) while photodesorption of $\mathrm{H}_{2}$ becomes more important at the PDR surface (Boschman et al. 2015), although the effect of increasing PAH size remains unclear.

Conclusive evidence for the presence of superhydrogenated PAHs in the ISM is still lacking. Spectral features attributed to aliphatic content of PAHs have been identified at 3.4, 6.9, and $7.25 \mu \mathrm{m}$ (Barker, Allamandola \& Tielens 1987; Bernstein, Sandford \& Allamandola 1996; Joblin et al. 1996; Sandford, Bernstein \& Materese 2013; Pilleri et al. 2015), and some of these have been detected in a range of galactic and magellanic cloud sources from young stellar objects (YSOs) over PDRs to the circumstellar environments of old stars (Habart et al. 2003; Goto et al. 2007; Sloan et al. 2007, 2014; Materese, Bregman \& Sandford 2017). Furthermore, it has been suggested that the change in the aromatic $7.7 \mu \mathrm{m}$ complex between different PAH classifications (Peeters et al. 2002; van Diedenhoven et al. 2004; Matsuura et al. 2014) may be attributable to changing importance of aliphatic material (Sloan et al. 2007, 2014; Boersma et al. 2008; Keller et al. 2008; Pino et al. 2008; Acke et al. 2010; Carpentier et al. 2012)

Here we present results showing the existence of stable configurations of superhydrogenated neutral coronene, and discuss the implications for molecular hydrogen formation in the ISM.

\section{METHODS}

\subsection{Experimental methods}

The experimental set-up and procedure for the temperature programmed desorption (TPD) measurements have been described in some detail previously (Thrower et al. 2012; Thrower et al. 2014; Skov et al. 2014, 2016). The TPD experiments were performed in a vacuum chamber under ultra-high vacuum (UHV) conditions, i.e. a base pressure of $1 \times 10^{-10}$ mbar. Coronene films were grown by evaporation of bulk coronene (Aldrich, sublimed, 99 per cent), from a home-built Knudsen cell-type source, operated at a temperature of $438 \mathrm{~K}$.

The coronene films were grown on a highly ordered pyrolitic graphite (HOPG, SPI grade 1) substrate. The sample was cleaved before mounting, and annealed to $1200 \mathrm{~K}$ in UHV before the experiments began in order to remove any contaminants from the surface. During coronene deposition the sample temperature was held at $289 \pm 5 \mathrm{~K}$. For each hydrogenation measurement, a coronene coverage of 1 monolayer (ML) was used. This was obtained by depositing sufficient coronene to build up 2-3 ML on the surface, followed by annealing to $397 \mathrm{~K}$ to desorb the multilayers, leaving a saturated ML. This allows for direct comparison of the temperature desorption yields between measurements.

The coronene films were then exposed to a beam of atomic hydrogen. The atoms were produced via thermal dissociation using a hot capillary source, similar to that described by Tschersich \& von Bonin (1998) and Tschersich (2000). The atom source was operated at a temperature of $2300 \mathrm{~K}$, at which it dissociates $\mathrm{H}_{2}$ (Air Liquide, N30, > 99.9 per cent). In order to cool down the hydrogen atoms, the $\mathrm{H}$ atom source was fitted with a twisted quartz nozzle, which collisionally cools the atoms to approximately $1000 \mathrm{~K}$ through multiple collisions with the nozzle wall. At this temperature no hydrogenation of the graphene basal plane is observed. The $\mathrm{H}$ atom flux at the sample position was determined to be $6( \pm 3) \times 10^{14}$ atoms $\mathrm{cm}^{-2} \mathrm{~s}^{-1}$ via calibration measurements of hydrogen uptake on the $\mathrm{Si}(100)$ surface as described in detail in Simonsen et al. (in preparation)

To measure the thermal desorption of superhydrogenated coronene molecules, the sample was heated in a controlled manner by $1 \mathrm{Ks}^{-1}$, using a PID controller (Lakeshore Model 340). The temperature of the sample was measured by a C-type thermocouple secured between the sample and sample holder, and the heating was achieved through electron bombardment of the back of the sample from a Ta filament.

The desorption products were measured by an Extrel CMS LLC quadrupole mass spectrometer. Singly ionized pristine coronene has $\mathrm{m} / z=300 \mathrm{amu}$ and singly ionized perhydrocoronene (fully superhydrogenated coronene) has $m / z=324 \mathrm{amu}$. Mass-to-charge ratios in the range 280-330 amu were monitored. The lower limit was chosen to detect fragmentation products where applicable, and the upper limit was chosen in order to establish a baseline, as no products with such masses should be present. The entire mass range could be scanned in $1 \mathrm{~s}$, thus providing good temperature resolution.

\subsection{Density functional theory calculations}

In parallel to the experimental work, extensive density functional theory (DFT) calculations were performed to identify the most stable structures and the possible hydrogenation pathways. The M06-2X hybrid-meta GGA density functional developed by Truhlar and coworkers (Zhao \& Truhlar 2008) was selected after a careful benchmark study on PAH chemistry (see the online Supplementary Material). This theory level is higher than those typically used in the past to investigate hydrogen adsorption on PAHs and graphene/graphite, and is seen to provide a much closer agreement with benchmark Coupled-Cluster calculations on coronene (Wang 


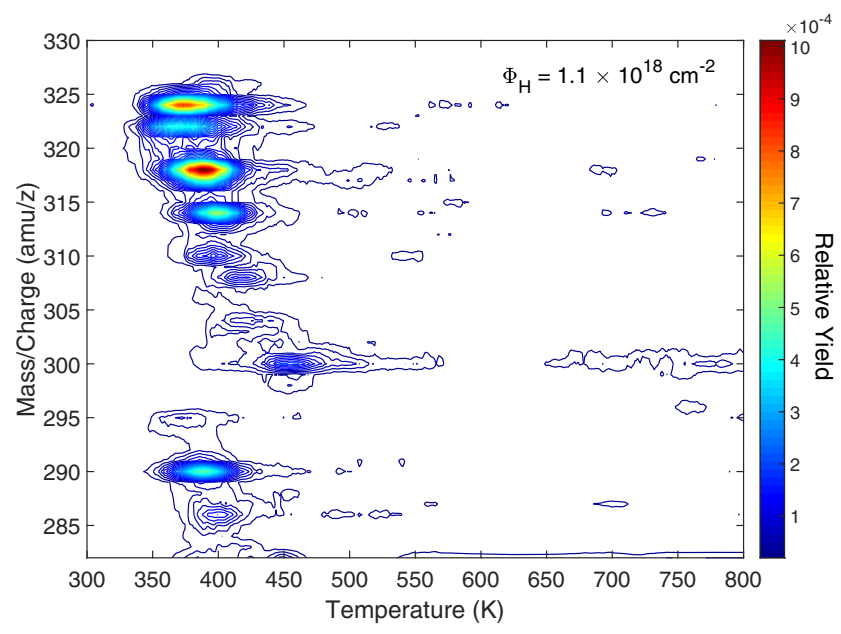

Figure 1. Contour plot showing the thermal desorption traces from coronene exposed to an atomic hydrogen fluence of $\Phi_{\mathrm{H}}=1.1 \times 10^{18} \mathrm{~cm}^{-2}$.

et al. 2012). Noteworthy, it gives adsorption barriers significantly higher than previously estimated, in both coronene and larger PAHs (Bonfanti, Achilli \& Martinazzo 2018).

The calculations described in the following were performed with the above-mentioned exchange-correlation functional in the spinunrestricted set-up, with the help of the Gaussian 16 code (Frisch et al. 2016). The 6-311G(d,p) atom-centred basis set was adopted throughout and tight convergence criteria were set on both the electronic and structural optimizations. Optimized structures were checked with harmonic frequency calculations.

Binding energies for the addition of $\mathrm{H}$ atoms were computed for a number of sites $i$ according to

$D_{i}^{n+1}=E_{*}^{n}+E_{H}-E_{i}^{n+1}$

where $E_{*}^{n}$ is the DFT energy of the most stable isomer with $n$ extra hydrogens, $E_{\mathrm{H}}$ is the energy of a hydrogen atom, and $E_{i}^{n+1}$ is the energy of the structure with an additional $\mathrm{H}$ at site $i$ (in the following, a star will be used to denote the most stable site, e.g. $D_{*}^{n} \equiv$ $\max _{i}\left\{D_{i}^{n}\right\}$ ). The plausible adsorption sites (typically 3-4 per step) were selected with the help of chemical intuition (Bonfanti et al. 2011) and Fukui's analysis (Contreras et al. 1999), and considered for adsorption at either side of the molecule, when meaningful. The sequence was continued by selecting at each step the structure with the largest binding energy, ${ }^{1}$ for a total of about 150 geometry optimizations. Energy barriers were computed for the most relevant cases only, by performing a transition-state search and subsequent frequency analysis.

\section{RESULTS}

Fig. 1 displays a contour plot showing the thermal desorption spectra for coronene exposed to an atomic hydrogen fluence of $\Phi_{\mathrm{H}}=1.1 \times$ $10^{18} \mathrm{~cm}^{-2}$ for all masses in the mass range 280-330 amu. High

\footnotetext{
${ }^{1}$ This does not guarantee that the structure is the lowest energy one for the given hydrogenation level. The reason is that it may well be possible (but hard to check or predict) that an 'energetic' structural optimization changes the energetic order between isomers because of, e.g. a conformational rearrangment, especially when they differ by few tenths of $\mathrm{eV}$ only. In a sense, the adopted procedure accounts for some possible kinetic impedements to the hydrogenation process.
}

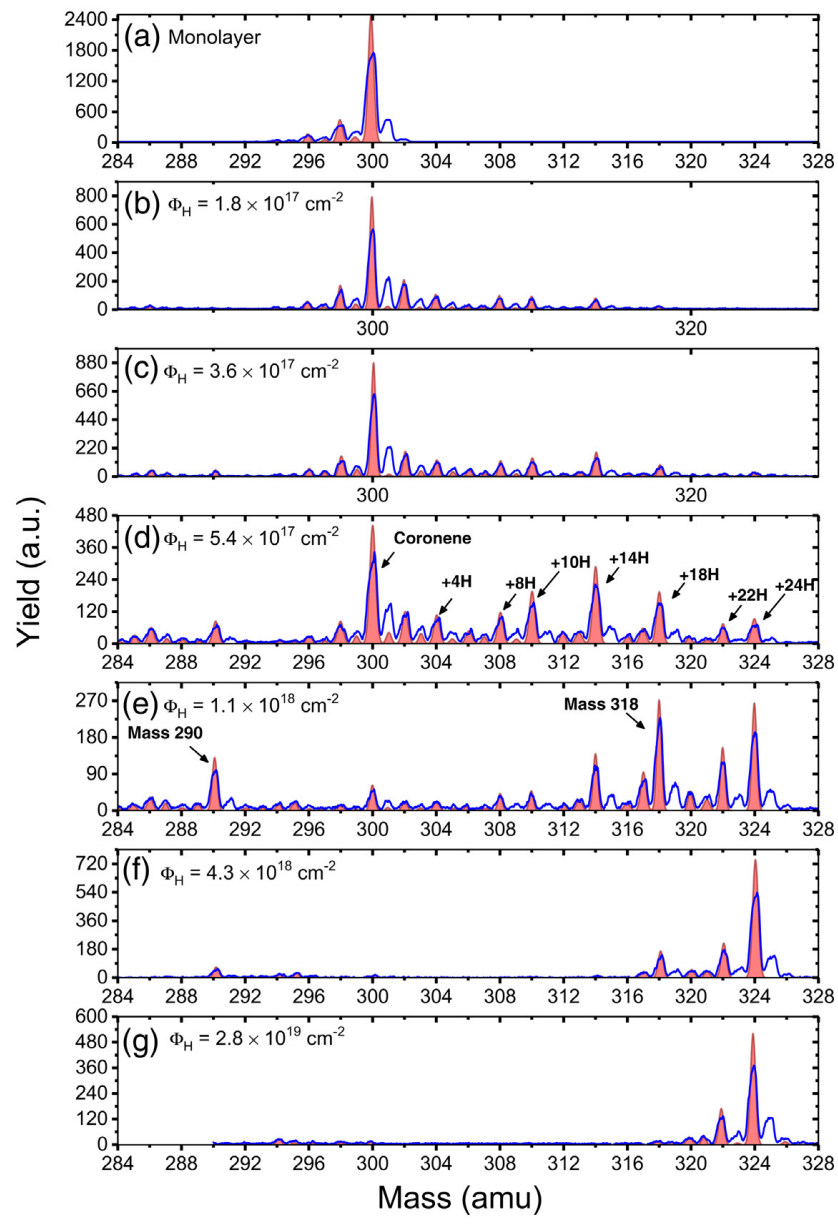

Figure 2. The blue lines show the mass distributions for coronene exposed to increasing atomic hydrogen fluences ranging from 0 to $\Phi_{\mathrm{H}}=2.8 \times$ $10^{19} \mathrm{~cm}^{-2}$. The red-shaded areas show how the peak structure would be in the absence of the ${ }^{13} \mathrm{C}$ isotope, which has a natural abundance of 1.109 per cent.

desorption yields in several masses lying above mass 300 indicate that a high degree of superhydrogenation has been reached. Even fully superhydrogenated coronene - perhydrocoronene - at 324 amu is observed confirming that hydrogenation can proceed to this upper limit. With the exception of the signal observed at $290 \mathrm{amu}$, the peak desorption temperature is observed to decrease as the mass of the molecule increases. This indicates a lower binding energy to the graphite substrate as more hydrogen is added to the molecule, as observed previously for the case of D-atom addition to coronene (Skov et al. 2016). While pristine coronene (300 amu) is observed to have a maximum desorption rate at $450 \mathrm{~K}$, fully superhydrogenated coronene (324 amu) is observed to desorb at a much lower temperature of $380 \mathrm{~K}$. The desorption behaviour of mass $290 \mathrm{amu}$ coincides with that of mass $318 \mathrm{amu}$, indicating that mass 290 may be a fragment of mass 318 after loss of e.g. a $\mathrm{C}_{2} \mathrm{H}_{4}$ molecule.

Integrating the individual thermal desorption traces results in mass distributions of superhydrogenated coronene for a given hydrogen dose. In Fig. 2 mass distributions are displayed for hydrogen fluences ranging from 0 to $\Phi_{\mathrm{H}}=2.8 \times 10^{19} \mathrm{~cm}^{-2}$. The blue line marks the experimental data. For the case of no hydrogen exposure (panel a), a large peak at 300 amu corresponding to pristine coronene is observed, with smaller peaks at slightly lower 


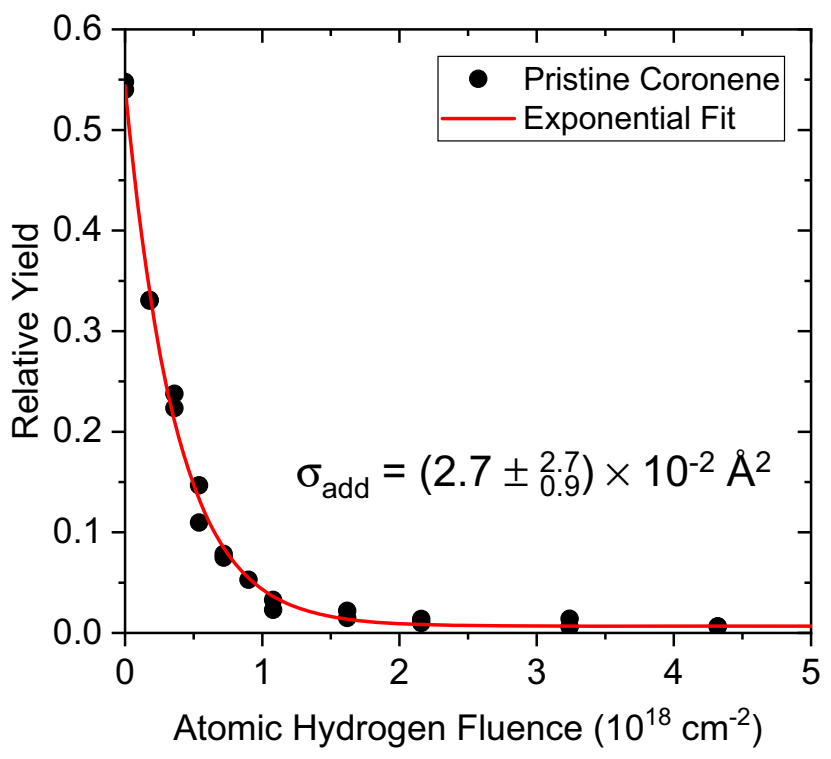

Figure 3. The experimentally derived relative yield in mass $300 \mathrm{amu}$ (pristine coronene) as a function of hydrogen fluence (dots). The red curve marks the best-fitting exponential decay.

masses due to hydrogen loss during the ionization step in the mass spectrometer. A small peak at $301 \mathrm{amu}$ is ascribed to the natural abundance of the heavier ${ }^{13} \mathrm{C}$ isotope in the coronene sample. As the dose time is increased, the peak at 300 amu becomes smaller and peaks at higher masses ascribed to superhydrogenated coronene begin to appear. Especially prominent peaks are observed at 304, $308,310,314,318,322$, and $324 \mathrm{amu}$, suggesting an increased stability for particular hydrogenation configurations. To facilitate comparison with theory, we also show how the mass spectra would look in the absence of the ${ }^{13} \mathrm{C}$ isotope. The red-shaded areas show how the peak structure would be if all ${ }^{13} \mathrm{C}$ istopes (assuming an abundance equal to the natural abundance of 1.109 percent) are converted to ${ }^{12} \mathrm{C}$. The main effect is apparant at small masses where the peak at 301 is drastically reduced. As a result the mass 302 peak now also stands out as a prominent peak. We also note that a drop in total signal is observed with $\mathrm{H}$ fluence - i.e. compare graph (a) and graph (g) in Fig. 2. This drop may be the result of fragmentation or chemical desorption (Minissale \& Dulieu 2014), where the energy released in the hydrogen addition reaction leads to desorption of the superhydrogeneted molecule and/or potentially also neighbour molecules.

Fig. 3 displays the evolution with atomic hydrogen exposure of the yield in mass $300 \mathrm{amu}$ - pristine coronene. An exponential decay with hydrogen fluence is observed and the cross-section for adding the first excess $\mathrm{H}$ atom to pristine coronene, $\sigma_{\text {add }}(0)$, can be found from this curve using the relation:

$I_{300}=I_{0} \exp \left(-\Phi_{\mathrm{H}} \sigma_{\mathrm{add}}(0)\right)+I_{\infty}$

where $I_{300}$ is the relative yield of pristine coronene for a given hydrogen fluence, $I_{0}$ is the relative yield of pristine coronene before hydrogen exposure, $I_{\infty}$ accounts for any background signal, and $\Phi_{\mathrm{H}}$ is the hydrogen fluence. A cross-section of $\sigma_{\text {add }}=2.7_{-0.9}^{+2.7} \times$ $10^{-2} \AA^{2}$ is found. Due to the decay in total signal during hydrogen exposure, this cross-section should be regarded as a lower limit.

In Fig. 4 the yield as a function of hydrogen exposure for the most prominent masses in Fig. 2 is displayed. As the fluence is increased, the yield of mass $300 \mathrm{amu}$ decreases exponentially, while masses
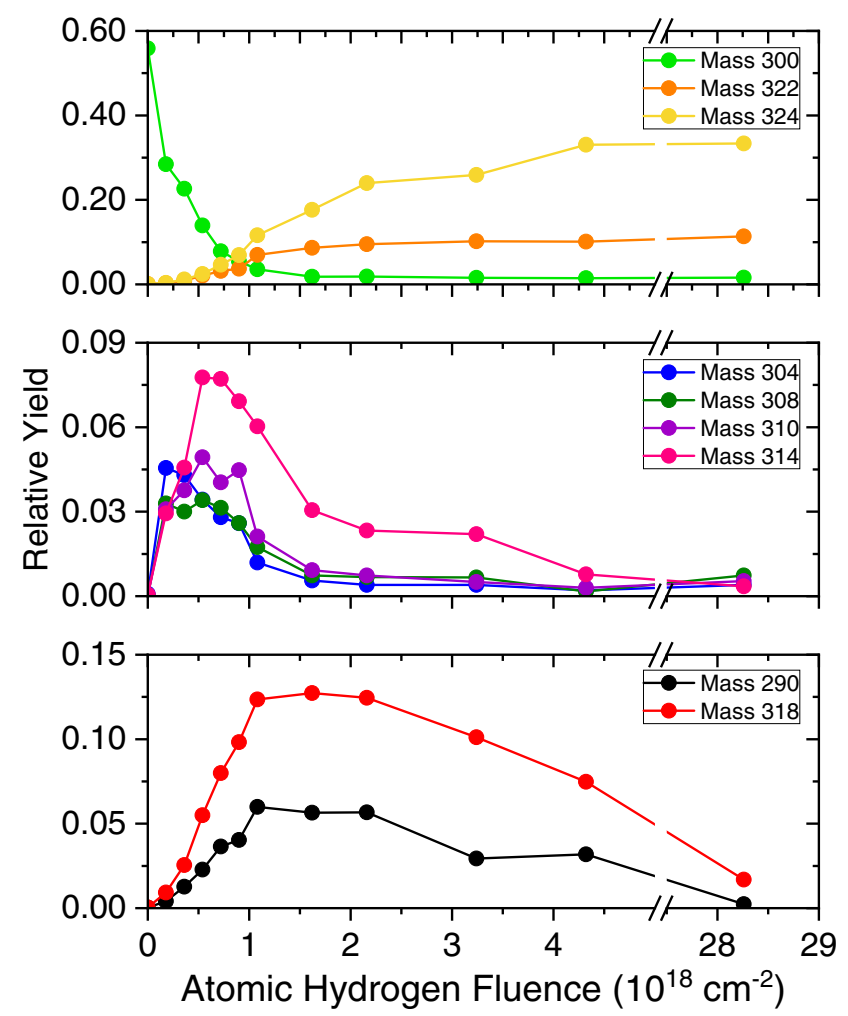

Figure 4. The relative yield of specific masses as a function of hydrogen fluence.

304, 308, 310, and 314 amu are observed to increase and then to subsequently decrease again at fluences above $\Phi_{\mathrm{H}}=1 \times 10^{18} \mathrm{~cm}^{-2}$, when even more highly superhydrogenated species begin to appear. The yield in 318 amu slowly starts to decrease at fluences above $\Phi_{\mathrm{H}}=2 \times 10^{18} \mathrm{~cm}^{-2}$ and disappears completely at very high hydrogen exposures. The yields of mass 322 and 324 amu slowly increase and dominate the spectrum at fluences above $\Phi_{\mathrm{H}}=1 \times$ $10^{18} \mathrm{~cm}^{-2}$. For the longest hydrogen exposures only mass 322 and 324 amu show significant yields indicating almost complete superhydrogenation. Furthermore, the evolution in the yields of masses 322 and 324 amu follows the same trend and displays similar thermal desorption behaviour (see Fig. 1). This indicates that the 322 amu peak may, at least in part, appear as a result of fragmentation of the fully superhydrogenated molecule (perhydrocoronene) in the mass spectrometer, through loss of $\mathrm{H}_{2}$.

The appearance of peaks at specific masses indicates that certain superhydrogenation configurations are more stable than others, and that when such stable configurations are reached, sizeable barriers for further additions exist. According to our calculations, the main hydrogenation sequence (i.e. the lowest energy pathway to perhydrocoronene) begins at an edge site before proceeding to center sites after a few $\mathrm{H}$ atom additions. Fig. 5 shows this lowest energy addition pathway. The energetics along this path is displayed in Fig. 6, which reports the sequential hydrogen adsorption energy $\left(D_{i}^{n}\right)$ as vertical bars. This figure shows an evident even-odd alternation arising from the larger exothermicity of radical-radical reactions, i.e. those forming even numbered $\mathrm{C}_{24} \mathrm{H}_{12+n}$ species. The amplitude of the oscillation of the adsorption energy of $\sim 2.5 \mathrm{eV}$ can be considered a rough measure of the $\pi$ bond strength, since the breaking of a $\pi$ bond is a necessary step only when forming odd numbered species (provided the molecule is in its ground-state, 


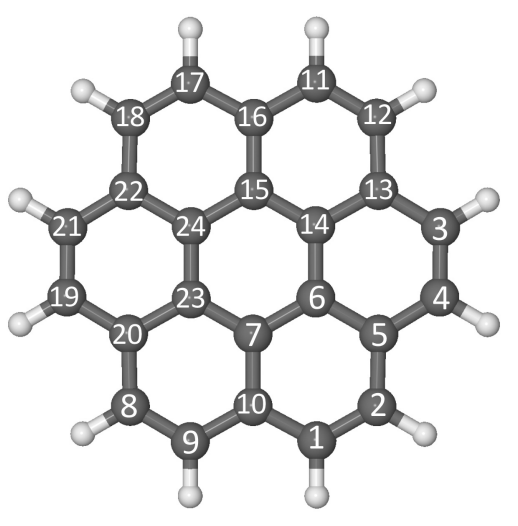

Figure 5. Main hydrogenation sequence of the coronene molecule. The numbers $j=1,2, \ldots, n$ indicate schematically the lowest energy isomer with $n$ extra hydrogen, $\mathrm{C}_{24} \mathrm{H}_{12}+n$.

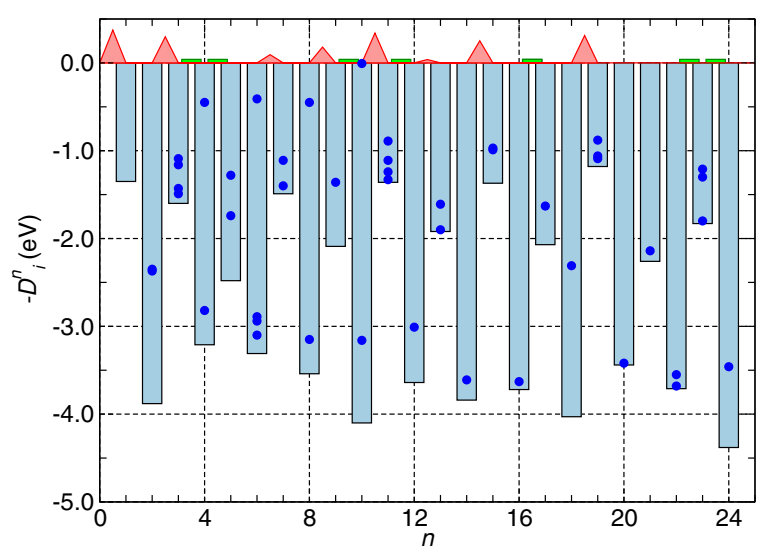

Figure 6. Hydrogen adsorption energy along the main sequence shown in Fig. 5 (light blue bars). The energy for alternative, slightly less stable hydrogenation configurations with the same number of excess $\mathrm{H}$ atoms is shown as dots. A red triangle between $n$ and $n+1$ represents the height of the barrier for sticking a $\mathrm{H}$ atom to the $n$-times hydrogenated coronene (multiplied by a factor of 2 to make it visible on the scale of the graph). Green bars denote situations where a barrierless adsorption path was found. A green bar is also used for the step $n=4 \rightarrow 5$, which presents a too little barrier $\left(E_{\mathrm{b}}=3.8 \mathrm{meV}\right)$ to be visible in the graph.

which is a doublet/singlet for $n$ odd/even). Since the adsorption barrier correlates well with $D_{i}^{n}$ (Evans \& Polanyi 1938; Casolo et al. 2009) - i.e. the larger the binding energy the smaller the barrier - a smaller binding energy is also indicative of a higher kinetic impediment to the addition reaction. The largest binding energies are found for coronene molecules with a specific number of extra hydrogens, $D_{*}^{n}=3.88,4.10,3.85,3.72,4.03,3.71$, and $4.38 \mathrm{eV}$, for $n=2,10,14,16,18,22$, and 24 , respectively.

To relate these findings to the experimentally observed abundances, one may argue as follows. Since $D_{*}^{n}$ is a measure of the stability of the species $\mathrm{C}_{24} \mathrm{H}_{12}+n$ against the desorption (or abstraction) of a $\mathrm{H}$ atom, and $\Delta D^{n}=D_{*}^{n}-D_{*}^{n+1}$ is a measure of the stability against further hydrogenation (it is, in fact, the energy of the reaction $2 \mathrm{C}_{24} \mathrm{H}_{12+n} \rightarrow \mathrm{C}_{24} \mathrm{H}_{12+n-1}+\mathrm{C}_{24} \mathrm{H}_{12+n+1}$ ), the combined index $\alpha=u D_{*}^{n}+v \Delta D^{n}$ (with $u, v$ some weights) should reflect the relative abundance of the species. Setting $u=v=1 / 2$ we find that the structures with $18,10,14$, and 2 extra $\mathrm{H}$ atoms (in decreasing order) are the most stable ones, with $\alpha=3.44,3.42,3.16$, and $3.08 \mathrm{eV}$, respectively. Adding to these the case $n=24-$ which
Table 1. Energy barriers (meV) for forming the $n$-times coronene superhydrogenated species $\left(\mathrm{C}_{24} \mathrm{H}_{12+n}\right)$, along the two pathways depicted in Figs 5 and $8(\mathrm{ND}=$ not determined).

\begin{tabular}{lcc}
\hline$n$ & Main sequence & Secondary sequence \\
\hline 1 & 187 & 187 \\
3 & 149 & 165 \\
4 & 0.0 & ND \\
5 & 3.8 & 159 \\
7 & 47 & 0.0 \\
9 & 90 & 74 \\
10 & 0.0 & 0.0 \\
11 & 170 & 178 \\
12 & 0.0 & ND \\
13 & 19 & 140 \\
15 & 126 & 0.0 \\
17 & 0.0 & 0.0 \\
19 & 153 & 155 \\
23 & 0.0 & ND \\
24 & 0.0 & 0.0 \\
\hline
\end{tabular}

has the largest $D_{*}^{n}$ along the entire pathway and, being last in the sequence, is indefinitely stable against further hydrogenation - we find a striking agreement with the thermal desorption spectra reported in Fig. 2. These conclusions are further corroborated by the heights of the hydrogen adsorption barriers (the energy barrier of the reactions $\mathrm{C}_{24} \mathrm{H}_{12+n}+\mathrm{H} \rightarrow \mathrm{C}_{24} \mathrm{H}_{12+n+1}$ ) that are found to be large for $n=0,2,10,14$, and 18 , with a smaller, but still significant barrier for $n=8$ and small (or even vanishing) barriers otherwise (see Fig. 6 and Table 1 for exact values).

Overall, hydrogen atom attachment to coronene can be rationalized with the help of some rules of thumb that should apply equally well to other PAHs. When closed-shell molecules are involved, e.g. $\mathrm{C}_{24} \mathrm{H}_{12+n}$ species with $n$ even, the factors driving the next addition are ' $\pi$-coordination' and ' $\pi$-hyperconjugation' (Bonfanti et al. 2011). The former determines the localization of frontier orbitals at the edges of the $\pi$ electronic system, enhancing the reactivity of those $s p^{2}$ sites that have the smallest number of $s p^{2}$ neighbours (e.g. two for the edge sites in pristine coronene or one for the site number 5 of Fig. 5 in the fourfold hydrogenated molecule). Hyperconjugation, on the other hand, discriminates between sites with the same $\pi$-coordination but different number of next-tonearest neighbours in the $\pi$ lattice. Hydrogen affinity increases with this number, as exemplified, for instance, by the cases of armchair edges (with no such second neighbours) and zig-zag ones (two second neighbours) that present markedly different hydrogenbinding energies, $D_{*} \sim 1.7$ and $2.8 \mathrm{eV}$, respectively (Bonfanti et al. 2018). For open-shell molecules (e.g. $\mathrm{C}_{24} \mathrm{H}_{12+n}$, with $n$ odd), on the other hand, the next addition is driven by the unpaired electron density and thus occurs at the so-called ortho and para positions where it mainly localizes through Pauli's resonance (Hornekær et al. 2006; Casolo et al. 2009) (again with a preference for sites with a small $\pi$-coordination).

Against this background, the exceptional stability of the hydrogenated species $\mathrm{C}_{24} \mathrm{H}_{12}+n$ with $n=2,10,14$, and 18 extra hydrogen can be traced back to additional electronic effects, namely to a 'residual aromaticity' that is left during the hydrogenation process. Indeed, as shown in Fig. 7, when $n=10,14$, and 18 the remaining $s p^{2}$ hybridized carbon atoms in the molecule still form a subsystem of three, two, or one aromatic rings, respectively, i.e. stable structural motifs that require extra energy for further hydrogen attachment to 
(a)

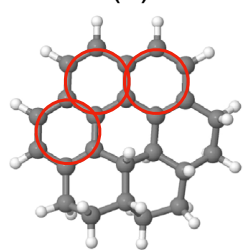

(c)

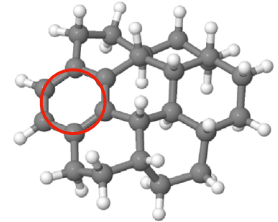

(b)

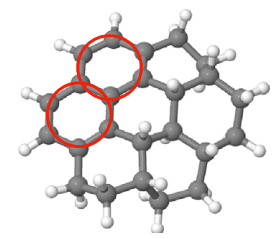

(d)

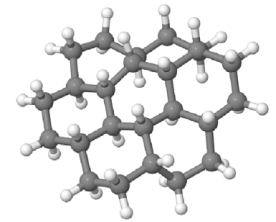

Figure 7. Superhydrogenated coronene molecule with 10, 14, 18, and 24 extra hydrogen atoms along the main sequence (panels a-d, respectively).

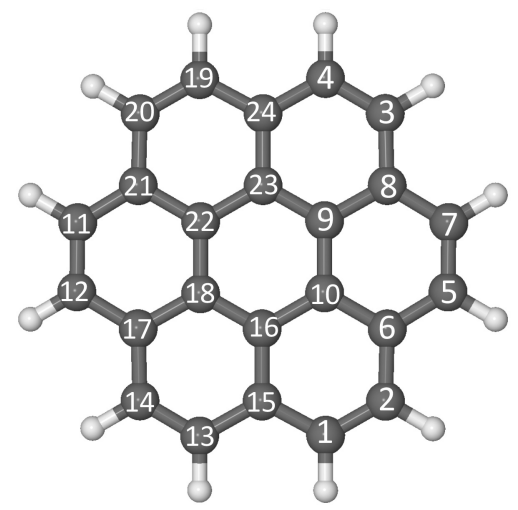

Figure 8. Alternative possible hydrogenation sequence of the coronene molecule to that of Fig. 5. It uses the second most stable site at the 3rd step and the most stable sites onward.

occur. The high stability of the structure with 24 extra $\mathrm{H}$, on the other hand, is owed to a complete saturation of the $\pi$-system.

Fig. 6 also shows, for each degree of superhydrogenation, the binding energy of a number of less stable hydrogenation configurations (see the online Supplementary material for details). Some of these values are very close to $D_{*}^{n}$, by less than $20 \mathrm{meV}-$ e.g. the ones relative to the $3 \mathrm{rd}$, 6th, or 11th steps - thereby suggesting that these values of $n$ may be branching points in the hydrogenation sequence. Therefore, an alternative hydrogenation pathway was investigated considering the second stable structure at the first branching point (3rd step), and keeping the lowest energy adsorption site onward. The resulting hydrogenation sequence is shown in Fig. 8, while the corresponding binding and barrier energies along the path are given in Fig. 9. The results parallel those found for the main sequence, and again identify $n=10,18$, and 24 as 'magic numbers'. In addition, the structure with $n=4$ is found to be more stable than in the previous path, in better agreement with the results of Fig. 2. However, a remarkably smaller binding energy (and a nondetectable barrier for further hydrogenation) results for the structure with 14 extra hydrogens, at odds with the thermal desorption spectra that show $\mathrm{C}_{24} \mathrm{H}_{26}$ accumulating during exposure (see Figs 2 and 4). With the same token, Fig. 9 also shows that the $n=12$ structure should accumulate during the exposure, but this is not observed in the mass traces.

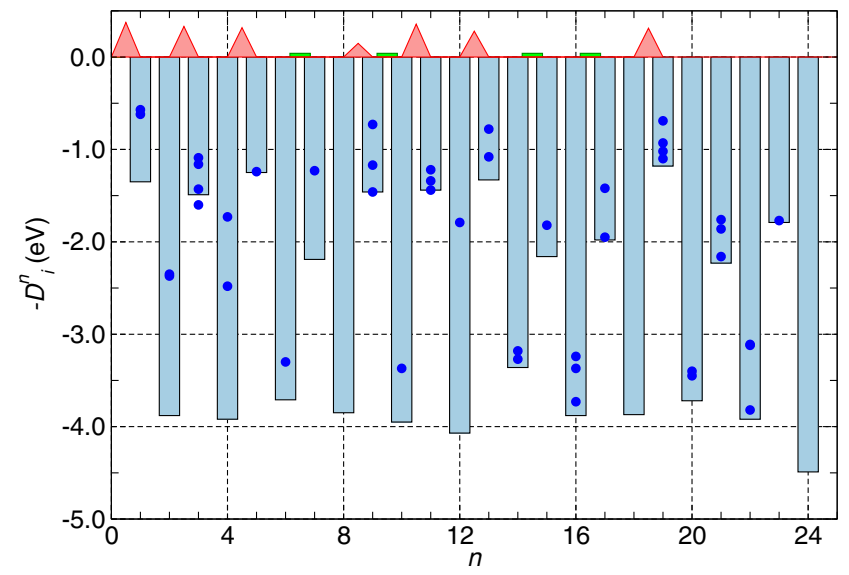

Figure 9. Hydrogen adsorption energy along the sequence shown in Fig. 8 (light blue bars). For each hydrogenation level, the energy for binding to alternative sites is shown as dots. Red triangles and green bars as in Fig. 6.

\section{CONCLUSIONS}

The presented combined thermal desorption spectroscopy measurements and DFT calculations reveal the most stable configurations in the superhydrogenation sequence of the PAH molecule coronene. Specifically experiments demonstrate the presence of stable configurations of superhydrogenated coronene at specific hydrogenation levels of $2,4,8,10,14,18$, and 24 extra hydrogen atoms. Such stable masses were not identified in previous experimental studies of coronene hydrogenation using $2000 \mathrm{~K}$ D atoms (Thrower et al. 2012). The appearance of stable masses observed here is ascribed to an increased sensitivity to reaction barriers for the lower temperature $(1000 \mathrm{~K}) \mathrm{H}$ atom beam. The experimental measurements also provide a lower limit for the cross-section for the first hydrogen atom addition to the neutral coronene molecule of $\sigma_{\text {add }}=2.7_{-0.9}^{+2.7} \times 10^{-2} \AA^{2}$. This cross-section is a factor of 20 lower than the cross-section previously found for addition of $2000 \mathrm{~K} \mathrm{D}$ atoms to coronene (Thrower et al. 2012). Both the energy difference and isotope effects are expected to contribute to the variation in cross-section, precluding a direct comparison until further experimental data are available. DFT calculations of binding energies and barrier heights, combined with considerations of the remnant aromaticity in superhydrogenated molecules, explain why some of superhydrogenation configurations are particularly stable. These considerations may be generalized to the superhydrogenation of other neutral PAH molecules and thus serve as a guideline for identifying which superhydrogenation states of neutral PAH molecules are expected to be the most abundant in interstellar environments favouring super-hydrogenation. Since the catalytic activity of these molecules for molecular hydrogen formation scales with the degree of superhydrogenation (Mennella et al. 2012), the inclusion of such stable superhydrogenation configurations in astrochemical models may give a more realistic picture of the contribution of superhydrogenated PAHs to interstellar chemistry.

\section{ACKNOWLEDGEMENTS}

The authors acknowledge financial support from The Danish Council for Independent Research (grant no. 5137-00049B), the CINECA award NEQRate under the ISCRA initiative, for the availability of high performance computing resources and support, The European Research Council (CoG GRANN, grant no. 648551), and EU under 
the Horizon2020 Marie Skodowska- Curie ITN EUROPAH (grant number 722346).

\section{REFERENCES}

Acke B., Bouwman J., Juhász A., Henning T., van den Ancker M. E., Meeus G., Tielens A. G. G. M., Waters L. B. F. M., 2010, ApJ, 718, 558

Allamandola L., Tielens A., Barker J., 1989a, ApJS, 71, 733

Allamandola L., Bregman J., Sandford S., Tielens A., Witteborn F., Wooden D., Rank D., 1989b, ApJ, 345, L59

Allamandola L. J., Hudgins D. M., Sandford S. A., 1999, ApJ, 511, L115

Andrews H., Candian A., Tielens A. G. G. M., 2016, A\&A, 595, A23

Bakes E. L. O., Tielens A. G. G. M., 1994, ApJ, 427, 822

Bakes E. L. O., Tielens A. G. G. M., 1998, ApJ, 499, 258

Barker J. R., Allamandola L. J., Tielens A. G. G. M., 1987, ApJ, 315, L61

Bauschlicher C. W., 1998, ApJ, 509, L125

Bauschlicher C. W., Peeters E., Allamandola L. J., 2009, ApJ, 697, 311

Bernstein M. P., Sandford S. A., Allamandola L. J., 2005, ApJS, 161, 53

Bernstein M. P., Sandford S. A., Allamandola L. J., 1996, ApJ, 472, L127

Bernstein M. P., Sandford S. A., Allamandola L. J., Gillette J. S., Clemett S. J., Zare R. N., 1999, Science, 283, 1135

Bernstein M. P., Elsila J. E., Dworkin J. P., Sandford S. A., Allamandola L. J., Zare R. N., 2002, ApJ, 576, 1115

Boersma C., Bouwman J., Lahuis F., Van Kerckhoven C., Tielens A., Waters L., Henning T., 2008, A\&A, 484, 241

Boersma C., Bauschlicher C., Allamandola L., Ricca A., Peeters E., Tielens A., 2010, A\&A, 511, A32

Bonfanti M., Casolo S., Ponti A., Tantardini G., Martinazzo R., 2011, J. Chem. Phys., 135, 164701

Bonfanti M., Achilli S., Martinazzo R., 2018, J. Phys. Condens. Matter, 30, 283002

Boschman L., Reitsma G., Cazaux S., Schlathölter T., Hoekstra R., Spaans M., Gonzalez-Magana O., 2012, ApJ, 761, L33

Boschman L., Cazaux S., Spaans M., Hoekstra R., Schlathölter T., 2015, A\&A, 579, A72

Bouwman J., Paardekooper D., Cuppen H., Linnartz H., Allamandola L., 2009, ApJ, 700, 56

Bouwman J., Mattioda A., Linnartz H., Allamandola L., 2011a, A\&A, 525, A93

Bouwman J., Cuppen H., Steglich M., Allamandola L., Linnartz H., 2011b, A\&A, 529, A46

Bregman J. D., Temi P., 2001, ApJ, 554, 126

Carpentier Y. et al., 2012, A\&A, 548, A40

Casolo S., Løvvik O. M., Martinazzo R., Tantardini G. F., 2009, J. Chem. Phys., 130, 054704

Cassam-Chenaï P., Pauzat F., Ellinger Y., 1994, AIP Conference Proceedings. Am. Inst. Phys., New York, p. 543

Cazaux S. et al., 2016, Sci. Rep., 6, 19835

Chiar J., Tielens A., Whittet D., Schutte W., Boogert A., Lutz D., Van Dishoeck E., Bernstein M., 2000, ApJ, 537, 749

Contreras R. R., Fuentalba P., Galvan M., Perez P., 1999, Chem. Phys. Lett., 304, 405

Duley W., 2006, Faraday Discuss., 133, 415

Elsila J. E., Hammond M. R., Bernstein M. P., Sandford S. A., Zare R. N., 2006, Meteorit. Planet. Sci., 41, 785

Evans M. G., Polanyi M., 1938, Trans. Faraday Soc., 34, 11

Frisch M. J. et al., 2016, Gaussian 16, Revision B.01

Gatchell M. et al., 2015, Phys. Rev. A, 92, 050702

Goto M. et al., 2007, ApJ, 662, 389

Habart E., Boulanger F., Verstraete L., des Forêts G. P., Falgarone E., Abergel A., 2003, A\&A, 397, 623

Habart E., Boulanger F., Verstraete L., Walmsley C., Des Forêts G. P., 2004, A\&A, 414, 531
Hornekær L. et al., 2006, Phys. Rev. Lett., 97, 186102

Hornekær L., Baurichter A., Petrunin V. V., Field D., Luntz A. C., 2003, Science, 302, 1943

Hudgins D. M., Bauschlicher C. W., Allamandola L., 2005, ApJ, 632, 316

Joblin C., Tielens A., Allamandola L., Geballe T., 1996, ApJ, 458, 610

Katz N., Furman I., Biham O., Pirronello V., Vidali G., 1999, ApJ, 522, 305

Keller L. D. et al., 2008, ApJ, 684, 411

Klærke B., Toker Y., Rahbek D. B., Hornekær L., Andersen L. H., 2013, A\&A, 549, A84

Léger A., d'Hendecourt L., 1985, A\&A, 146, 81

Leger A., Puget J., 1984, A\&A, 137, L5

Le Page V., Snow T. P., Bierbaum V. M., 2009, ApJ, 704, 274

Manico G., Raguni G., Pirronello V., Roser J., Vidali G., 2001, ApJ, 548, L253

Materese C. K., Bregman J. D., Sandford S. A., 2017, ApJ, 850, 165

Matsuura M. et al., 2014, MNRAS, 439, 1472

Mennella V., Hornekær L., Thrower J., Accolla M., 2012, ApJ, 745, L2

Minissale M., Dulieu F., 2014, J. Chem. Phys., 141, 014304

Peeters E., Hony S., Van Kerckhoven C., Tielens A., Allamandola L., Hudgins D., Bauschlicher C., 2002, A\&A, 390, 1089

Pilleri P., Joblin C., Boulanger F., Onaka T., 2015, A\&A, 577, A16

Pino T. et al., 2008, A\&A, 490, 665

Rapacioli M., Cazaux S., Foley N., Simon A., Hoekstra R., Schlathölter T., 2018, Phys. Chem. Chem. Phys., 20, 22427

Rasmussen J. A., Henkelman G., Hammer B., 2011, J. Chem. Phys., 134, 164703

Rauls E., Hornekær L., 2008, ApJ, 679, 531

Reitsma G., Boschman L., Deuzeman M. J., González-Magaña O., Hoekstra S., Cazaux S., Hoekstra R., Schlathölter T., 2014, Phys. Rev. Lett., 113, 053002

Sandford S. A., Bernstein M. P., Materese C. K., 2013, ApJS, 205, 8

Skov A., Thrower J., Hornekær L., 2014, Faraday Discuss., 168, 223

Skov A. W., Andersen M., Thrower J. D., Jørgensen B., Hammer B., Hornekær L., 2016, J. Chem. Phys., 145, 174708

Sloan G. et al., 2007, ApJ, 664, 1144

Sloan G. et al., 2014, ApJ, 791, 28

Thrower J. D. et al., 2012, ApJ, 752, 3

Thrower J. D., Friis E. E., Skov A. L., Jørgensen B., Hornekær L., 2014, Phys. Chem. Chem. Phys., 16, 3381

Tielens A. G., 2008, ARA\&A, 46, 289

Tielens A. G. G. M., 2013, Rev. Mod. Phys., 85, 1021

Tschersich K., 2000, J. Appl. Phys., 87, 2565

Tschersich K., von Bonin V., 1998, J. Appl. Phys., 84, 4065

van Diedenhoven B., Peeters E., Van Kerckhoven C., Hony S., Hudgins D., Allamandola L., Tielens A., 2004, ApJ, 611, 928

Wakelam V., Herbst E., 2008, ApJ, 680, 371

Wang Y., Qian H., Morokuma K., Irle S., 2012, J. Phys. Chem. A, 116, 7154

Wolf M., Kiefer H. V., Langeland J., Andersen L. H., Zettergren H., Schmidt

H. T., Cederquist H., Stockett M. H., 2016, ApJ, 832, 24

Zhao Y., Truhlar G., 2008, Theor. Chem. Acc., 120, 215

\section{SUPPORTING INFORMATION}

Supplementary data are available at MNRAS online.

\section{SI_Jensen2019.pdf}

Please note: Oxford University Press is not responsible for the content or functionality of any supporting materials supplied by the authors. Any queries (other than missing material) should be directed to the corresponding author for the article.

This paper has been typeset from a $\mathrm{T}_{\mathrm{E}} \mathrm{X} / \mathrm{L} \mathrm{T}_{\mathrm{E}} \mathrm{X}$ file prepared by the author. 